\title{
Bilateral Cataract Development and Pupillary Block Glaucoma Following Implantable Collamer Lens
}

Ohoud Owaidhah ${ }^{1}$, Huda Al-Ghadeer ${ }^{2}$

\begin{abstract}
Aim and objective: We report the first case of bilateral cataract formation and pupillary block glaucoma and high intraocular pressure (IOP) following implantable Collamer lens (ICL) implantation that resulted in advanced visual field loss.

Background: The patient who underwent bilateral ICL implantation can develop bilaterally elevated IOP and an anterior subcapsular cataract with altitudinal visual field defect.

Case description: A 38-year-old man with high myopia presented for routine follow-up status post bilateral phakic ICL placement. The visual acuity was reduced due to an anterior subcapsular cataract and elevated IOP in both eyes with advanced glaucomatous visual field defects. The patient was treated with topical glaucoma medications. The left eye underwent same-day phakic ICL explanation and cataract surgery to prevent further visual field loss.

Conclusion: Cataract and glaucoma are serious complications after phakic ICL implantation; therefore, regular postoperative monitoring may prevent advanced visual impairment.

Clinical significance: The use of a phakic intraocular lens for the correction of myopia may result in complications. As a measure is to reduce such complications, refractive surgeons preferred using phakic posterior chamber intraocular Collamer lens for the correction of myopia.

Keywords: Acute angle closure glaucoma, Cataract, Glaucoma, Implantable collamer lens, Intraocular pressure, Pupillary block.

Journal of Current Glaucoma Practice (2021): 10.5005/jp-journals-10078-1309
\end{abstract}

\section{BACKGROUND}

Refractive surgery using a phakic posterior chamber intraocular Collamer lens (ICL) (Visian ICL ${ }^{\mathrm{T}}$; Staar Inc., Monrovia, CA, USA) is approved by the US Food and Drug Administration (FDA) for the correction of myopia.'

The first toric ICL (STAAR Surgical, Monrovia, CA, USA) was implanted in 1999 by Thomas Neuhann in Munich (unpublished data) in 2002. The first implant in North America was reported by Gimbel and Zremb. ${ }^{2}$ Since these initial cases, numerous ICLs have been implanted worldwide. However, there are some complications of phakic intraocular lenses (pIOLs) including trauma to the crystalline lens, high intraocular pressure (IOP), retinal detachment, postoperative infection, corneal endothelial loss, and cataract formation. ${ }^{1,3}$

To our knowledge, we report the first case of bilateral cataract formation and pupillary block glaucoma, and high IOP following ICL implantation that resulted in advanced visual field loss.

\section{Case Description}

A 38-year-old man with high myopia and astigmatism presented for a refractive surgery evaluation. The patient had no prior history of glaucoma or ocular trauma. The preoperative refraction was- $9.00-2.25 \times 85^{\circ}$ in the right eye $-9.00-3.00 \times 85^{\circ}$ in the left eye. The patient had a stable refractive history and open angles on gonioscopy. Dilated fundus exam showed myopic changes, tilted discs with a C/D ratio of 0.4 in both eyes. The anterior chamber depth was $>3.0 \mathrm{~mm}$ and the endothelial cell count was $2,587 \mathrm{cell} / \mathrm{mm}^{2}$ in the right eye and $2,512 \mathrm{cell} / \mathrm{mm}^{2}$ in the left eye with normal morphology. The preoperative evaluation included a comprehensive slit-lamp examination which was unremarkable. The high refractive error and the volume of tissue removal precluded
1Department of Glaucoma, King Khaled Eye Specialist Hospital, Riyadh, Kingdom of Saudi Arabia

${ }^{2}$ Department of Anterior Segment, King Khaled Eye Specialist Hospital, Riyadh, Kingdom of Saudi Arabia

Corresponding Author: Huda Al-Ghadeer, Department of Anterior Segment, King Khaled Eye Specialist Hospital, Riyadh, Kingdom of Saudi Arabia, Phone: +966 114821234 Ext. 2500, e-mail: hghadeer@ kkesh.med.sa

How to cite this article: Owaidhah O, Al-Ghadeer H. Bilateral Cataract Development and Pupillary Block Glaucoma Following Implantable Collamer Lens. J Curr Glaucoma Pract 2021;15(2):91-95.

Source of support: Nil

Conflict of interest: None

treatment with excimer laser surgery. After a thorough discussion, the patient opted for ICL implantation.

A single PI was performed in each eye at 12 o'clock with a neodymium:YAG (Nd:YAG) laser 4 weeks preoperatively. The Visian ICLs were selected based on sulcus diameters measured by the Pentacam (Oculus Optikgerate GmbH, Wetzlar, Germany).

$[-12.00+2.00 \times 171$ OD-13.00-3.00 $\times 174$ OS $]$.

The eye was prepared in a sterile fashion and the two drops of topical anesthetic were placed. A lid speculum placed for maximum corneal exposure. A 3.2-mm peripheral temporal clear corneal wound was created for implantation of the ICL (Visian ICL ${ }^{\mathrm{TM}}$ model ICMV4b, Staar Inc., Monrovia, CA, USA). There were no complications during surgery in both eyes.

A few hours after surgery, the patient presented with moderate ocular pain in the right eye, his IOP was $48 \mathrm{~mm} \mathrm{Hg}$ in the right eye and $19 \mathrm{~mm} \mathrm{Hg}$ in the left eye (measured with Goldmann applanation 
tonometry), the anterior chamber was moderately shallow with mild corneal edema. The ICLs were well positioned in the posterior chamber.

The PI was partially patent and the ICLs were in place with a high vault. Retained viscoelastic material was diagnosed. The patient has been prescribed acetazolamide tablets $250 \mathrm{mg}$ qid PO, timolol maleate eye drops (0.5\%) bid, and brimonidine tartrate eye drops $0.15 \%$ bid. After 24 hours, the IOP decreased to $8 \mathrm{~mm} \mathrm{Hg}$ and the cornea cleared, so the patient was discharged with instructions to start dilating drops, topical Alphagan (Allergan plc, Dublin, Ireland), and Cosopt (dorzolamide/timolol) 2\%/0.5\% (MSD, Kenilworth, NJ, USA) for the right eye.

On the second postoperative day, the patient presented with moderate diffuse corneal edema with an IOP of $29 \mathrm{~mm} \mathrm{Hg}$ in the right eye. The dilating drops were discontinued, augmentation of the PI was performed with a neodymium:YAG (Nd:YAG) laser. A few hours after the YAG PI, the IOP stabilized at $9 \mathrm{~mm} \mathrm{Hg}$ and the PI was patent but the anterior chamber was relatively shallow, so the dilating drops were prescribed again. Right eye pupillary block was diagnosed. The patient was seen for another two visits postoperatively, with the well-controlled IOP in the right eye; hence, the topical brimonidine tartrate was discontinued.

Four months postoperatively, the patient presented with early lens changes in the right eye (Fig. 1A), and IOP was controlled on topical Cosopt. Visual acuity was $20 / 40$ and endothelial cell count was within normal limits. The patient underwent ICL implantation in the left eye and surgery was uneventful. The left eye remained stable for the early postoperative visits. The patient lost to follow-up and presented 4 months after surgery with 20/40 visual acuity in the right eye and 20/60 in the left eye. The IOPs were 19 and 45 $\mathrm{mm} \mathrm{Hg}$ in the right and left eyes, respectively. The Pls were patent bilaterally with high vault and moderately shallow AC, cataract (Fig. 1B), and shallow cupping of the left eye with a C/D ratio of 0.64 and 0.8 in right and left eyes, respectively. The patient was using only Cosopt for the right eye so stat medications were given to reduce IOP in the left eye resulting in a decrease to $22 \mathrm{~mm} \mathrm{Hg}$.

Baseline glaucoma investigations indicated a central corneal thickness was 512 and $516 \mu$ in the right and left eyes, respectively. Humphrey's visual field testing (24-2 SITA-standard) was relatively normal in the right eye and showed a superior altitudinal defect in the left eye (Fig. 2). OCT (RTVue; Optovue, Inc., Fremont, CA, USA) imaging confirmed the clinical findings (Fig. 3).
Given the presence of a visually significant cataract and elevated IOP, the phakic ICL in the left eye was removed concurrently with cataract surgery to prevent further visual field loss. Postoperatively, the patient's IOP remained controlled on topical glaucoma medications, and his best-corrected visual acuity improved to $20 / 20$ in this eye.

AS-OCT of the right and left eye (Figs 4A and B) showing an absence of excess ICL vaulting, shallow anterior chamber. Ultrasound biomicroscopy (UBM) demonstrating direct contact (white arrow) between ICL and the posterior iris in the left eye with the shallow anterior chamber and anterior vaulting of ICL consistent with pupillary block following ICL implantation (Fig. 4C).

\section{Discussion}

This case describes a patient who underwent bilateral ICL implantation with bilaterally elevated IOP and an anterior subcapsular cataract and altitudinal visual field defect in the left eye. A previous study of plOLs has reported anterior subcapsular opacities in 11 cases $(2.1 \%){ }^{4}$ However, two $(0.4 \%)$ cases required pIOL explanations with cataract extraction and IOL implantation at 1 -year follow-up. ${ }^{4}$ At 3 years postoperatively, 14 eyes $(2.7 \%)$ had anterior subcapsular opacities. ${ }^{5}$

Sanders and Vukich ${ }^{6}$ compared the incidence of lens opacities and clinically significant cataracts with two ICL designs. The V3 model was implanted in 87 eyes, and the V4 model was implanted in 523 eyes. They found that the incidence of anterior subcapsular opacities was significantly higher with the V3 model compared with the V4 model (12.6 vs $2.9 \%$ ). Furthermore, $9.2 \%$ of eyes with the V3 model and $0.8 \%$ of eyes with the V4 model had clinically significant cataracts. ${ }^{6}$ The higher incidence of lens opacities and cataracts was due to a greater proportion of eyes with poor vaulting in the V3 group.

Sanders ${ }^{7}$ found that $6-7 \%$ of eyes developed anterior subcapsular opacities 7 years after ICL implantation. However, only $1-2 \%$ of eyes that received an ICL, progress to clinically significant cataracts, particularly older patients and those with very high myopia and older model ICLs as in our patient. ${ }^{7-9}$ Of the eyes that require $\mathrm{pIOL}$ explanation and phacoemulsification with posterior IOL implantation, $47 \%$ were patients younger than 40 years and $53 \%$ were patients 40 years or older. Of these eyes, $14 \%$ had less than $-10.50 \mathrm{D}$ of myopia, $19 \%$ had
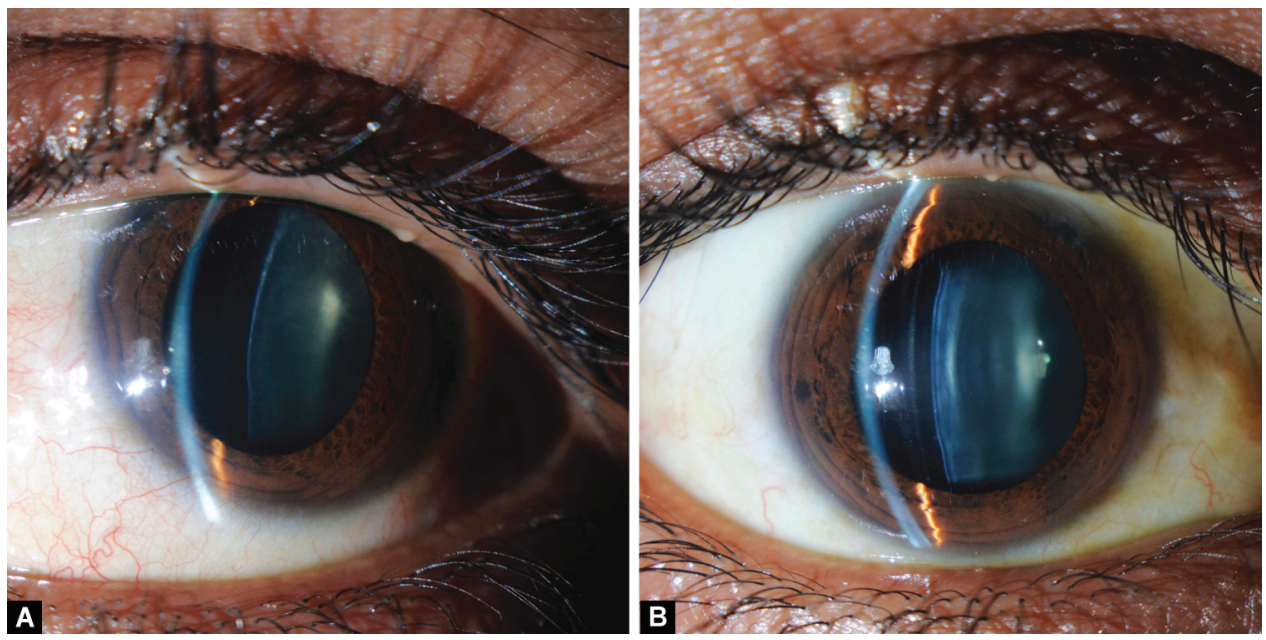

Figs $1 \mathrm{~A}$ and B: Slit lamp photograph shows anterior subcapsular cataract 

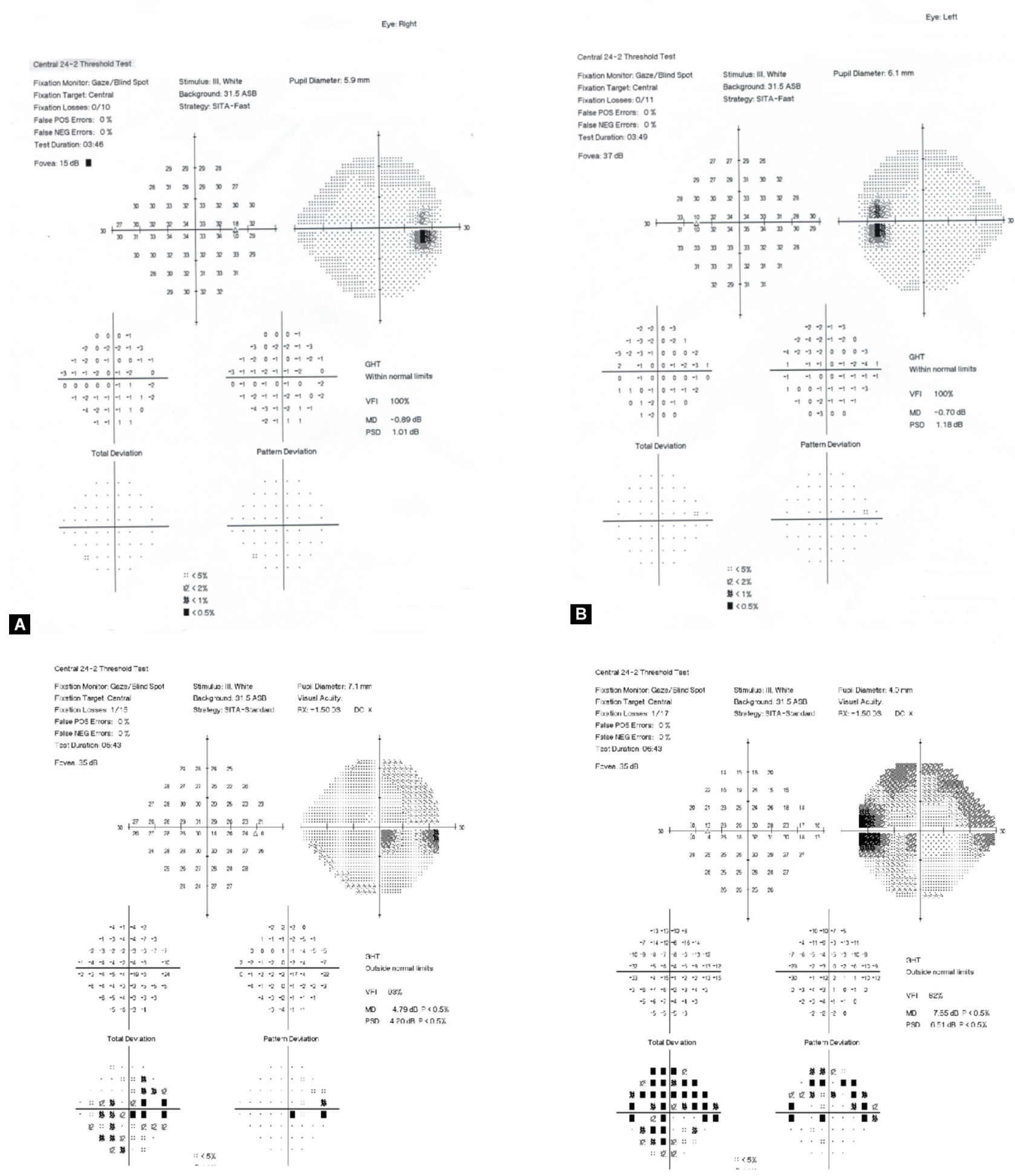

C

D

Figs 2A to D: (A and B) Preoperative visual field test (24-2 SITA-standard) shows a normal field in the right eye and in the left eye; (C and D) Preoperative visual field test (24-2 SITA-standard) shows a relatively normal field in the right eye and a superior altitudinal defect in the left eye

between $-10.50 \mathrm{D}$ and $-13.50 \mathrm{D}$ of myopia, and $67 \%$ had more than $-13.50 \mathrm{D}$ of myopia. ${ }^{8}$

The relationship between vaulting and cataract formation has been extensively studied. ${ }^{6-10}$ Gonvers et al. ${ }^{10}$ found that central vaulting $>90 \mathrm{~mm}$ appears to protect the crystalline lens from cataract formation. However, higher central vaulting (approximately $150 \mathrm{~mm}$ ) is recommended to avoid contact between the pIOL and the crystalline lens because vault distance showed a slight decrease over time. They also found that cataracts developed more commonly in older patients than in younger patients. 
Right /OD

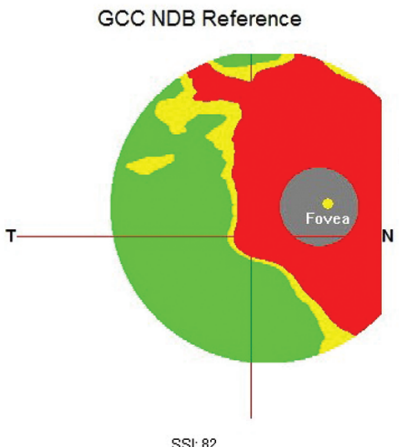

SSI: 82

Left/OS

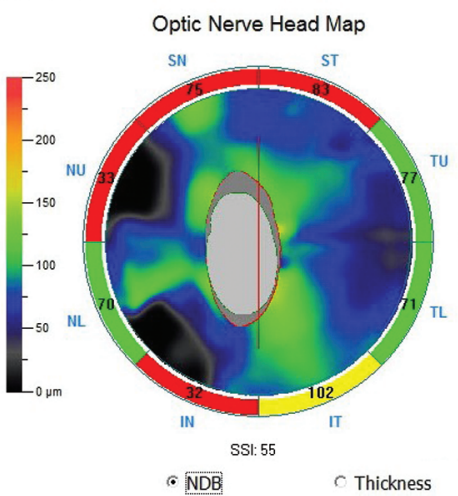

\section{ONH/GCC OU Report}
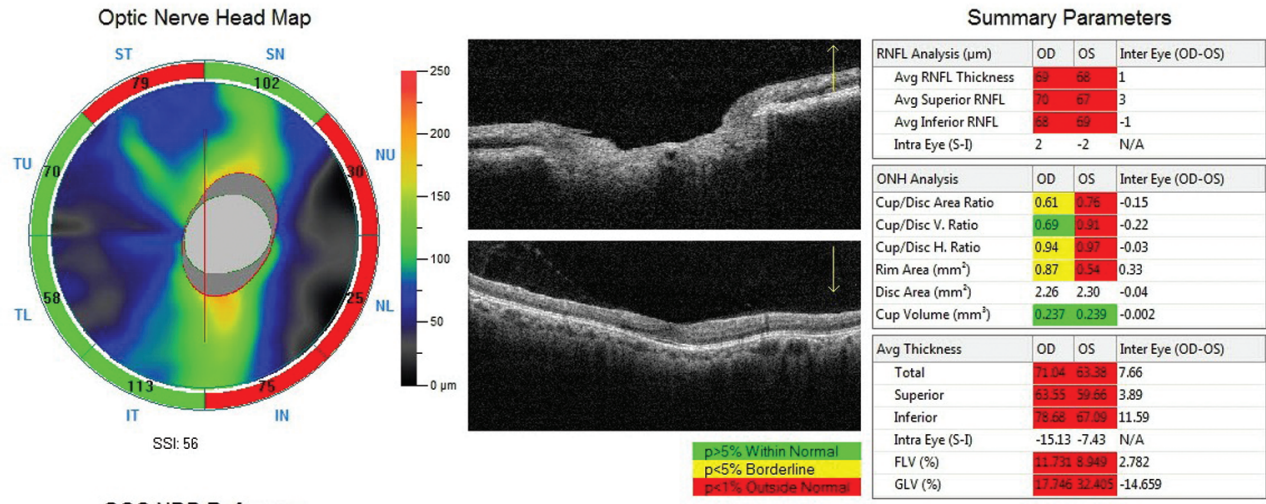

TSNIT NDB Reference
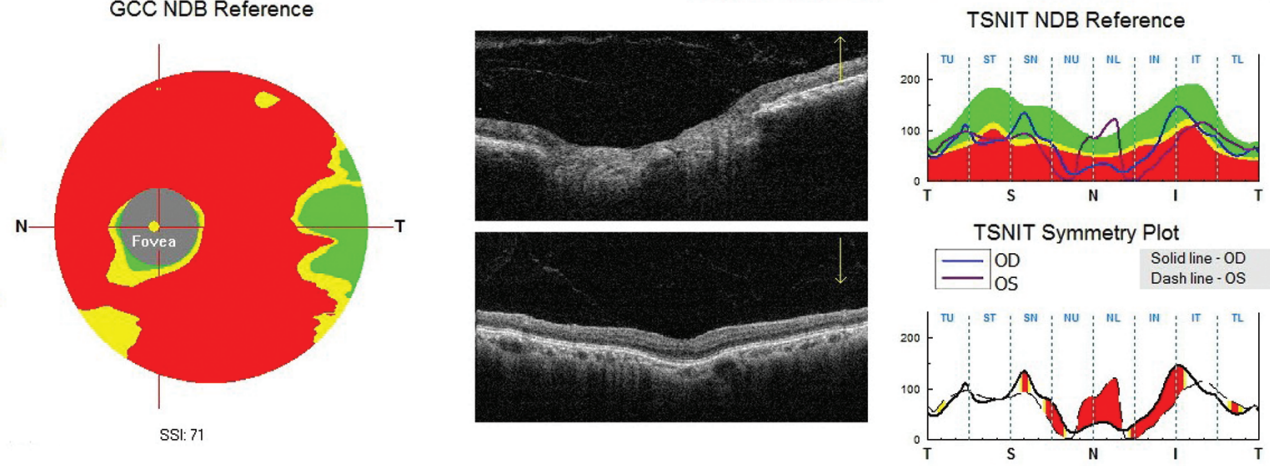

Fig. 3: Postoperative spectral-domain optical coherence tomography (SD-OCT) of both eyes shows more RNFL thickness reduction in the nasal portion of the optic nerve in OU. The mean RNFL average for the OD was $69 \mu \mathrm{m}$ and for the OS $68 \mu \mathrm{m}$. The ganglion cell complex thickness analysis was reduced in OS more than OD
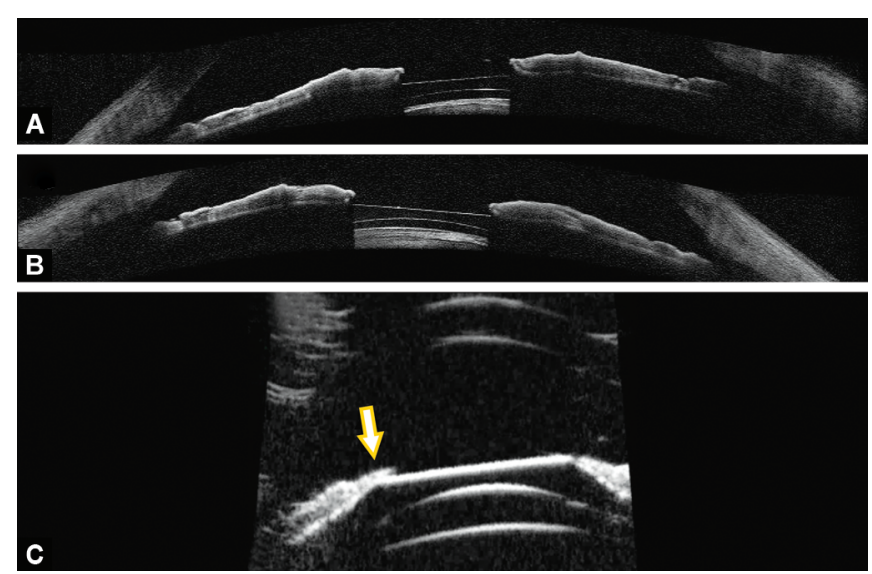

Figs 4A to C: (A and B) AS-OCT of the right eye and the left eye shows an absence of excess ICL vaulting, shallow anterior chamber; $(C)$ Ultrasound biomicroscopy (UBM) demonstrates direct contact (white arrow) between ICL and the posterior iris in the left eye with the shallow anterior chamber and anterior vaulting of $\mathrm{ICL}$ consistent with pupillary block following ICL implantation

Increased IOP after ICL implantation has been previously reported and can occur at the early or late postoperative period. ${ }^{11}$ The causes are attributed to retained viscoelastic material, steroid response, secondary angle closure, and high ICL vaulting. ${ }^{3}$ Although the mechanism of angle closure in cases of ICL implantation has not been fully explained, one of the proposed mechanisms is acute angle closure with a pupillary block which may be due to contact between the iris margin and ICL optic, preventing the flow of aqueous with subsequent iris bombe and elevated IOP. ${ }^{12}$ This form of pupillary block can be prevented with PIs, ${ }^{12}$ two Pls are recommended in case one gets occluded. ${ }^{12}$

The other mechanism (angle-closure without pupillary block) can be due to the large ICL diameter. ${ }^{3}$ This mechanism is likely due to the central vaulting which creates a separation between the crystalline lens and the ICL centrally, but there can be contacted in the midperiphery blocking the normal circulation of aqueous humor. This mechanism cannot be reversed with $\mathrm{PI}$ and topical cycloplegia is required. Cycloplegia increases the diameter of the ciliary sulcus and reduces ICL vaulting. ${ }^{10}$ To resolve the pupillary block, ICL explanation is warranted. Our patient experienced angle closure with a pupillary block in the right eye that was successfully managed with PI augmentation. The left eye had angle closure (due to oversized ICL) which was accompanied by lens changes, uncontrolled IOP, and visual field changes, the decision to remove the ICL and to perform phacoemulsification with IOL implantation helped to relieve the attack, improve the visual acuity, and stabilize the visual field.

\section{Conclusion}

Although the incidence of cataracts is low after plOL implantation, the rate of cataracts is higher in older patients and in those with high refractive errors. 


\section{Clinical Significance}

Cataract and glaucoma are serious complications after phakic ICL implantation; therefore, regular postoperative monitoring may prevent advanced visual impairment.

The use of a plOL for the correction of myopia may result in complications. As a measure is to reduce such complications, refractive surgeons preferred using phakic posterior chamber intraocular Collamer lens for the correction of myopia.

Presented as a poster in the ESCRS 2019.

\section{References}

1. Clara Y, Patel CK, Momont AC, et al. Advanced pigment dispersion glaucoma secondary to phakic intraocular collamer lens implant. Am J Ophthalmol Case Rep 2018;10:65-67. DOI: 10.1016/j.ajoc.2018. 01.046.

2. Sanders DR, Schenider D, Martin D, et al. Toric implantable collamer lens for moderate to high myopic astigmatism. Ophthalmology 2007;114(1):54-61. DOI: 10.1016/j.ophtha.2006.08.049.

3. Almalki S, Abubaker A, Alsabaani N, et al. Causes of elevated intraocular pressure following implantation of phakic intraocular lens for myopia. Int Ophthalmol 2016;36(2):259-265. DOI: 10.1007/ s10792-015-0112-4.

4. The Implantable Contact Lens in Treatment of Myopia (ITM) Study Group. U.S. Food and Drug Administration clinical trial of the implantable contact lens for moderate to high myopia. Ophthalmology 2003;110(2):255-266. DOI: 10.1016/S01616420(02)01771-2.
5. ICL in Treatment of Myopia (ITM) Study Group. United states food and drug administration clinical trial of the implantable collamer lens (ICL) for moderate to high myopia; three-year follow-up. Ophthalmology 2004;111(9):1683-1692. DOI: 10.1016/j.ophtha.2004.03.026.

6. Sanders DR, Vukich JA. Incidence of lens opacities and clinically significant cataracts with the implantable contact lens: comparison of two lens designs. J Refract Surg 2002;18(6):673-682. DOI: 10.3928/1081-597X-20021101-03.

7. Sanders DR. Anterior subcapsular opacities and cataract 5 years after surgery in the visian implantable collamer lens: FDA trial. J Refract Surg 2008;24(6):566-570. DOI: 10.3928/1081597X-20080601-04.

8. Alfonso JF, Lisa C, Vega LF, et al. Prevalence of cataract after collagen copolymer phakic intraocular lens implantation for myopia hyperopia, and astigmatism. J Cataract Refract Surg 2015;41(4): 800-805. DOI: 10.1016/j.jcrs.2014.07.039.

9. Mudr HK. Incidence of cataract following implantation of a posterior chamber phakic lens ICL (implantable collamer lens)-long-term results. Čes a slovOftal 2017;73:87-93.

10. Gonvers M, Bornet C, Othenin-Girard P. Implantable contact lens for moderate to high myopia; relationship of vaulting to cataract formation. J Cataract Refract Surg 2003;29(5):918-924. DOI: 10.1016/ s0886-3350(03)00065-8.

11. Senthil S, Choudhari NS, Vaddavalli PK, et al. Etiology and management of raised intraocular pressure following posterior chamber phakic intraocular lens implantation in myopic eyes. PloS One 2017;12(2):e0172929. DOI: 10.1371/journal.pone.0172929.

12. McCaughey MV, Mifflin T, Fenzl CR, et al. Pseudophacomorphic glaucoma along with pupillary block after visian ${ }^{\mathrm{TM}}$ implantable collamer lens implantation for high myopia. Open J Ophthalmol 2014;4(4):107-111. DOI: 10.4236/ojoph.2014.44017. 